\title{
Anomaly-mediated SUSY breaking model retrofitted for naturalness
}

\author{
Howard Baer, ${ }^{1, *}$ Vernon Barger, ${ }^{2, \dagger}$ and Dibyashree Sengupta ${ }^{1, \hbar}$ \\ ${ }^{1}$ Department of Physics and Astronomy, University of Oklahoma, Norman, Oklahoma 73019, USA \\ ${ }^{2}$ Department of Physics, University of Wisconsin, Madison, Wisconsin 53706, USA
}

(Received 2 February 2018; published 31 July 2018)

\begin{abstract}
Anomaly-mediated supersymmetry breaking (AMSB) models seem to have become increasingly implausible due to 1) difficulty in generating a Higgs mass $\left.m_{h} \sim 125 \mathrm{GeV}, 2\right)$ typically unnatural superparticle spectra characterized by a large superpotential mu term, and 3) the possibility of a winolike lightest supersymmetry particle as dark matter now seems to be excluded. In the present paper, we propose some modifications to the paradigm model which solve these three issues. Instead of adding a universal bulk scalar mass to avoid tachyonic sleptons, we add distinct Higgs and matter scalar soft masses which then allow for light Higgsinos. To gain accord with the measured Higgs mass, we also include a bulk trilinear soft term. The ensuing natural generalized AMSB model then has a set of light Higgsinos with mass nearby the weak scale $m(W, Z, h) \sim 100 \mathrm{GeV}$ as required by naturalness, while the winos populate the several hundred $\mathrm{GeV}$ range and gluinos and squarks occupy the multi-TeV range. For LHC searches, the wino pair production followed by decay to the same-sign diboson signature channel offers excellent prospects for discovery at the high luminosity LHC along with Higgsino pair production leading to soft dileptons plus jet(s) + MET. A linear $e^{+} e^{-}$collider operating above the Higgsino pair production threshold should be able to distinguish the AMSB gaugino spectra from unified or mirage unified scenarios. Dark matter is expected to occur as a Higgsino-like weakly interacting massive particle plus axion admixture.
\end{abstract}

DOI: 10.1103/PhysRevD.98.015039

\section{INTRODUCTION}

The discovery of $D$-branes in superstring models in the 1990s [1] ushered in new avenues for particle physics model building. In the case of supersymmetry (SUSY), this was exemplified initially with the advent of models where the dominant contribution to soft SUSY breaking Lagrangian parameters originated from violations of the superconformal anomaly, in what became known as anomaly-mediated SUSY breaking models (AMSBs) $[2,3]$. The AMSB contributions to soft SUSY breaking terms are always present in gravity mediation, but since they occur at loop level, they are usually suppressed compared to tree-level contributions and hence had previously been mostly neglected. Randall and Sundrum (RS) constructed an extra-dimensional scenario where the AMSB soft term contributions were expected to be the

\footnotetext{
*baer@nhn.ou.edu

†barger@pheno.wisc.edu

‡Dibyashree.Sengupta-1@ ou.edu
}

Published by the American Physical Society under the terms of the Creative Commons Attribution 4.0 International license. Further distribution of this work must maintain attribution to the author(s) and the published article's title, journal citation, and DOI. Funded by SCOAP ${ }^{3}$. dominant or nearly dominant terms. The initial idea was that the visible sector, usually assumed to be the minimal supersymmetric Standard Model (MSSM), would be located on one three-brane extending through an assumed extra-dimensional spacetime, while SUSY breaking would occur on a different brane. Thus, the SUSY breaking sector was in fact sequestered, or separated from the visible sector brane within the extra-dimensional spacetime. This setup suppressed tree-level SUSY breaking soft terms in the visible sector. But since gravity propagates in the bulk, the entire extra-dimensional spacetime, the anomaly-mediated contributions could dominate the visible sector soft terms.

The AMSB gaugino masses were calculated to be proportional to the corresponding gauge group beta functions times the gravitino mass

$$
M_{i}=\frac{\beta_{i}}{g_{i}} m_{3 / 2}
$$

with $\beta_{i}=\frac{g_{i}^{3}}{16 \pi^{2}} b_{i}, b_{i}=(6.6,1,-3)$, and where $i$ labels the gauge group. At the weak scale, taking into account the running gauge coupling values, one would expect gaugino masses in the ratio $M_{1}: M_{2}: M_{3} \sim 3.3: 1:-9$ so that the winos are the lightest of the weak-scale gauginos. This is in contrast to models with unified gaugino masses where the bino occurs as the lightest gaugino. The lightest neutral 
wino was then typically assumed to be the lightest SUSY particle (LSP) in AMSB with striking consequences for collider and dark matter signatures [4-6].

In addition, in AMSB, the soft breaking scalar masses were computed to be

$$
m_{\tilde{f}}^{2}=-\frac{1}{4}\left\{\frac{d \gamma}{d g} \beta_{g}+\frac{d \gamma}{d f} \beta_{f}\right\} m_{3 / 2}^{2},
$$

where $\beta_{f}$ is the beta function for the corresponding superpotential Yukawa coupling and anomalous dimension $\gamma=\partial \ln Z / \partial \ln \mu$ with $Z$ the wave function renormalization constant and $\mu$ is the running energy scale. The AMSB contribution to trilinear soft SUSY breaking terms is given by

$$
A_{f}=\frac{\beta_{f}}{f} m_{3 / 2}
$$

where $f$ is the corresponding Yukawa coupling.

For some assumed value of gravitino mass $m_{3 / 2} \sim 50-100 \mathrm{TeV}$, all the AMSB soft terms are comparable to each other with values near to the weak scale as required by phenomenology. An annoyance is that the slepton masses turn out to be tachyonic with negative mass squared leading to an electric charge breaking minimum for the scalar potential. It was suggested by RS [2] that additional bulk contributions to scalar masses, which are comparable to the AMSB contributions, could be present to alleviate this problem. ${ }^{1}$ An assortment of other solutions to the negative slepton mass problem were also devised [11].

To gain concrete phenomenological predictions for AMSB at colliding beam and dark matter detection experiments, a minimal AMSB model (mAMSB) was devised wherein a common bulk contribution $m_{0}^{2}$ was appended to all AMSB scalar mass-squared values $[5,6] .{ }^{2}$ Once the weak-scale soft terms were determined, then the superpotential $\mu$ term was tuned so as to maintain the measured value of the $Z$-boson mass via the scalar potential

\footnotetext{
${ }^{1}$ In this paper (as in many others on the topic of AMSB phenomenology [5-10]), we parametrize our ignorance of the origin of the bulk soft terms by merely introducing them without a specific theory as to how they arise. Indeed, in the absence of experimental guidance as to the structure of the sequestered sector, most specific models of how the bulk terms arise are likely to be wrong.

${ }^{2} \mathrm{~A}$ point of obvious concern is that additional bulk soft terms may reintroduce the SUSY flavor problem [12] for which AMSB models might offer a solution. In this paper, we do not offer a solution to the SUSY flavor problem which might arise from extra bulk soft term contributions. Instead, we regard the lack of large flavor changing neutral currents in experiment as an interesting constraint for sequestered-sector model builders to address. Here, we merely adopt flavor-preserving bulk soft terms into our effective SUSY theory which are at least consistent with phenomenology (as do many other papers on AMSB phenomenology) [2,5-10].
}

minimization conditions. Thus, the parameter space of the mAMSB model was given by

$$
m_{0}, \quad m_{3 / 2}, \quad \tan \beta, \quad \operatorname{sign}(\mu) .
$$

Expectations for LHC searches within the mAMSB construct have been presented in Refs. [7,8]. Searches for direct chargino pair production in mAMSB with disappearing tracks from long-lived but ultimately unstable winolike charginos [4] have been presented by ATLAS [13].

The minimal AMSB model has provided a beautiful and compelling framework for new physics searches. It has been especially appreciated for containing solutions to the SUSY flavor problem (since the sfermions of each generation acquire common masses) and the gravitino problem (since gravitinos are so heavy that they decay much more quickly than the $\mathrm{TeV}$-scale gravitinos which are expected in usual supergravity (SUGRA) models). While winolike weakly interacting massive particles (WIMPs) are thermally underproduced in the mAMSB model, it was hypothesized by Moroi and Randall [14] that nonthermal WIMP production from, for instance, decay of light moduli fields could augment the relic abundance of dark matter and bring its mass abundance into accord with measured values.

While the mAMSB model is a well-motivated and beautiful construct, recently, it has suffered several setbacks on the phenomenological front:

(i) The first of these was the discovery of the Higgs boson at a mass value $m_{h} \simeq 125 \mathrm{GeV}$. In the mAMSB model, the trilinear soft terms given by Eq. (3) are generally not large enough to lift the predicted value of $m_{h}$ into the $125 \mathrm{GeV}$ range unless sparticle masses are very heavy-in the vicinity of tens of $\mathrm{TeV}$ [15-17]. Such heavy sparticle masses exacerbate the so-called little hierarchy problem which arises from the growing mass gap between the measured value of the weak scale and the sparticle mass scale.

(ii) The second setback arises from nonobservation of sparticles at the CERN LHC. While one solution to this issue is to simply posit that the mAMSB sparticles are heavier than experimental limits, this also makes the theory increasingly unnatural [18] and hence increasingly implausible.

(iii) A third setback arose on the dark matter front. In mAMSB, where a winolike WIMP is expected to comprise the dark matter, the model has come into conflict with new stringent limits from direct and indirect dark matter detection experiments. Searches for WIMPs at underground noble liquid experiments-which test the spin-independent (SI) direct detection (DD) rate-apparently exclude about half the remaining mAMSB parameter space [19]. Meanwhile, indirect WIMP detection (IDD) searches - via observation of gamma rays arising from WIMP-WIMP annihilation into hadrons 
followed by e.g., $\pi^{0} \rightarrow \gamma \gamma$ decay — have placed severe limits on wino dark matter. The Fermi-LAT/MAGIC Collaboration [20], via a search for gamma rays from dwarf spheroidal galaxies, now seems to require $m($ wino $) \gtrsim 700 \mathrm{GeV}$. Along with this, the HESS experiment [21], from 254 hours (10 years) of observation of continuum gamma rays arising from the Galactic center, now requires $m$ (wino) $\gtrsim$ $1200 \mathrm{GeV}$. If Sommerfeld enhancement effects are included in the WIMP-WIMP annihlation rate, then winolike WIMPs seem to be excluded over their entire mass range [19,22,23]. At first sight, such limits from IDD dark matter searches would seem to exclude models like mAMSB with winolike WIMP dark matter. ${ }^{3}$

To expand upon the fine-tuning/naturalness issue, we adopt here the most conservative fine-tuning measure, $\Delta_{\text {EW }}[25,26]$. The quantity $\Delta_{\text {EW }}$ measures how well the weak-scale MSSM Lagrangian parameters match the measured value of the weak scale. By minimizing the MSSM weak-scale scalar potential to determine the Higgs field vacuum expectation values (vevs), one derives the wellknown expression relating the Z-boson mass to the SUSY Lagrangian parameters:

$$
\begin{aligned}
\frac{m_{Z}^{2}}{2}= & \frac{m_{H_{d}}^{2}+\Sigma_{d}^{d}-\left(m_{H_{u}}^{2}+\Sigma_{u}^{u}\right) \tan ^{2} \beta}{\tan ^{2} \beta-1}-\mu^{2} \simeq-m_{H_{u}}^{2} \\
& -\Sigma_{u}^{u}\left(\tilde{t}_{1,2}\right)-\mu^{2} .
\end{aligned}
$$

Here, $\tan \beta=v_{u} / v_{d}$ is the ratio of Higgs field vacuum expectation values and the $\Sigma_{u}^{u}$ and $\Sigma_{d}^{d}$ contain an assortment of radiative corrections, the largest of which typically arise from the top squarks. Expressions for the $\Sigma_{u}^{u}$ and $\Sigma_{d}^{d}$ are given in the Appendix of Ref. [26]. Thus, $\Delta_{\mathrm{EW}}$ compares the maximal contribution on the rhs of Eq. (5) to the value of $m_{Z}^{2} / 2$. If the rhs terms in Eq. (5) are individually comparable to $m_{Z}^{2} / 2$, then no unnatural fine-tunings are required to generate $m_{Z}=91.2 \mathrm{GeV}^{4}$

The main requirements for low electroweak fine-tuning $\left(\Delta_{\mathrm{EW}} \lesssim 30\right)^{5}$ are the following:

\footnotetext{
${ }^{3}$ A possibility which avoids these constraints consists of mixed wino/axion dark matter [24].

${ }^{4}$ Other measures include $\Delta_{B G} \equiv \max _{i}\left|\frac{p_{i}}{m_{Z}^{2}} \frac{\partial m_{Z}^{2}}{\partial p_{i}}\right|$, where $p_{i}$ are fundamental parameters of the theory [27]. In a theory where all soft terms are interdependent (such as AMSB or SUGRA or gaugemediated SUSY breaking (GMSB) ), $\Delta_{B G}$ reduces to $\Delta_{\text {EW }}$ [18]. Sometimes $\Delta_{H S} \equiv \delta m_{h}^{2} / m_{h}^{2}$ is used [28] where $\delta m_{h}^{2} \sim$ $-\frac{3 f_{t}^{2}}{8 \pi^{2}}\left(m_{Q_{3}}^{2}+m_{U_{3}}^{2}+A_{t}^{2}\right) \ln \left(\Lambda^{2} / m_{S U S Y}^{2}\right)$ with $f_{t}$ the top Yukawa coupling, $\Lambda$ is as high as $m_{\mathrm{GUT}}$, and $m_{\mathrm{SUSY}} \sim 1 \mathrm{TeV}$. This measure has been oversimplified by neglecting the $m_{H_{u}}^{2}$ contribution to its own running so as not to allow for radiatively driven naturalness, where large high-scale soft terms are driven by radiative corrections to natural values at the weak scale $[29,30]$.

${ }^{5}$ The onset of fine-tuning for $\Delta_{\mathrm{EW}} \gtrsim 30$ is visually displayed in Ref. [31].
}

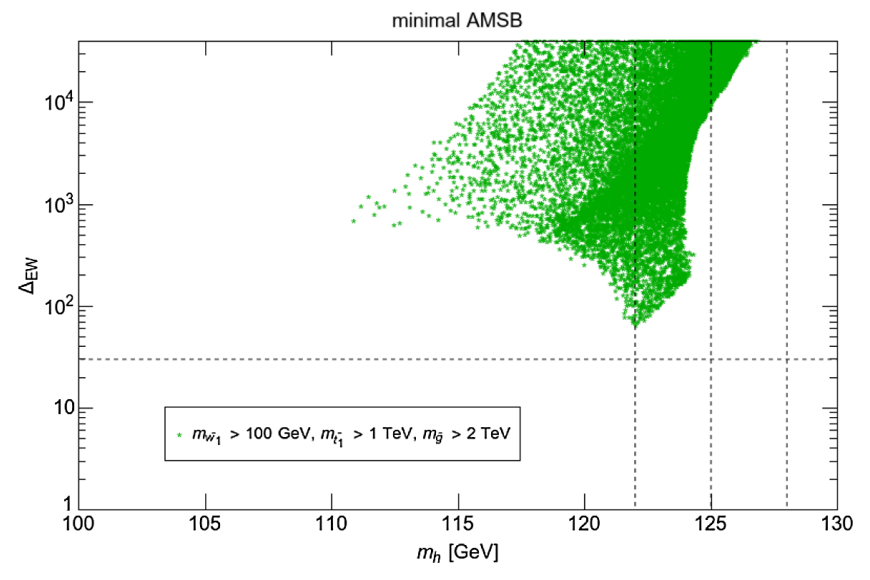

FIG. 1. Plot of points from a scan over mAMSB parameter space in the $\Delta_{\mathrm{EW}}$ vs $m_{h}$ plane.

(i) $|\mu| \sim 100-300 \mathrm{GeV}[32,33]$ (the lighter the better), where $\mu \gtrsim 100 \mathrm{GeV}$ is required to accommodate LEP2 limits from chargino pair production searches.

(ii) $m_{H_{u}}^{2}$ is driven radiatively to small-not largenegative values at the weak scale $[25,26]$.

(iii) The top-squark contributions to the radiative corrections $\Sigma_{u}^{u}\left(\tilde{t}_{1,2}\right)$ are minimized for $\mathrm{TeV}$-scale highly mixed top squarks [25]. This latter condition also lifts the Higgs mass to $m_{h} \sim 125 \mathrm{GeV}$. For $\Delta_{\text {EW }} \lesssim 30$, the lighter top squarks are bounded by $m_{\tilde{t}_{1}} \lesssim 3 \mathrm{TeV}[26,31]$.

(iv) The gluino mass, which feeds into the stop masses at one loop and hence into the scalar potential at twoloop order, is bounded by $m_{\tilde{g}} \lesssim 6 \mathrm{TeV}[26,31]$.

In Fig. 1, we show the results of a scan over the mAMSB model parameter space in the $\Delta_{\mathrm{EW}}$ vs $m_{h}$ plane. We use ISAJET7.88 [34] to generate the mAMSB spectra. We have scanned over:

(i) $m_{0}: 1-10 \mathrm{TeV}$,

(ii) $m_{3 / 2}: 80-1000 \mathrm{TeV}$,

(iii) $\tan \beta: 4-58$,

with $\mu>0$. $^{6}$

From Fig. 1, we see that the minimal value of $\Delta_{\mathrm{EW}}$ occurs around 100 so that indeed the model is fine-tuned in the electroweak sector at least at the $\sim 1 \%$ level. The lowest $\Delta_{\text {EW }}$ points occur at $m_{3 / 2} \sim 100 \mathrm{TeV}$ where $m_{\tilde{g}} \sim 2 \mathrm{TeV}$, just beyond the current LHC $m_{\tilde{g}}$ mass limit [35].

\footnotetext{
${ }^{6} \mathrm{~A}$ point of concern may be that the soft terms (aside from gaugino masses) may emerge as bulk rather than AMSB dominated. Indeed, with $m_{3 / 2}$ ranging from $80-1000 \mathrm{TeV}$, for the slepton masses, the required bulk contributions range from 280-3500 GeV just to avoid becoming tachyonic (so their soft terms of course must be bulk dominated to at least cancel off the negative AMSB contribution). Our range of bulk mass contributions typically lies within a factor of several of the required minimal bulk masses required to avoid tachyons. These are comparable to [9] or smaller than [10] the range of bulk soft terms explored in other papers.
} 
While many of these points have $m_{h} \sim 122 \mathrm{GeV}$, to gain $m_{h} \sim 125 \mathrm{GeV}$, the value of $\Delta_{\mathrm{EW}}$ jumps to $\gtrsim 6000$.

To improve upon this situation, in this paper, we present a retrofitted phenomenological AMSB model which is a generalization of mAMSB and which addresses the three issues discussed above. Indeed, in the original RS paper [2], the authors actually advocated for the modifications we present here. It was only when some simplifications were implemented in the original minimal AMSB model that these features were abandoned $[5,6]$. The two generalizations to mAMSB include the following:

(1) independent bulk contributions $m_{H_{u}}^{2}($ bulk) and $m_{H_{d}}^{2}(b u l k)$ to the soft SUSY breaking Higgs masses as opposed to matter scalar bulk masses $m_{0}^{2}(1,2)$ (for first/second generation matter scalars) and $m_{0}^{2}(3)$ (for third generation matter scalars) and

(2) inclusion of bulk contributions $A_{0}$ to the trilinear soft terms.

These two modest changes in the AMSB model will allow each of the three issues above to be circumvented. However, we will also see that the anticipated collider phenomenology and dark matter expectations will be very different. After bringing the model into accord with the measured Higgs mass and naturalness, the LSP will no longer be a winolike neutralino but instead a Higgsino-like neutralino. If we posit that the SUSY $\mu$ problem is solved via the Kim-Nilles mechanism [36] (a supersymmetrized version of the Dine-Fischler-Srednicki-Zhitnitsky (DFSZ) axion model [37] which allows for $\mu \ll m_{\text {soft }}$ ), then dark matter is expected to consist of an axion plus Higgsino-like WIMP admixture [38].

In Sec. II, we make explicit our modified AMSB soft term formulas. We also present aspects of the anticipated natural AMSB spectra where now the LSP is expected to be a Higgsino-like neutralino but where the lightest gaugino is still expected to be winolike. Since the model can now be rendered natural, we dub the resultant model nAMSB, or natural anomaly mediation, to distinguish it from the previously explored minimal AMSB model. We present some benchmark spectra and a nAMSB model line. In Sec. III, we discuss consequences of the nAMSB model for collider and dark matter searches. In Sec. IV, we summarize and present our conclusions.

\section{NATURAL ANOMALY-MEDIATED SUSY BREAKING MODEL}

\section{A. Soft terms for nAMSB}

In this section, we propose several modifications of the mAMSB model which will allow for naturalness along with a Higgs mass $m_{h} \simeq 125 \mathrm{GeV}$.

For gaugino masses, we maintain the usual formulas:

$$
M_{1}=\frac{33}{5} \frac{g_{1}^{2}}{16 \pi^{2}} m_{3 / 2}
$$

$$
\begin{gathered}
M_{2}=\frac{g_{2}^{2}}{16 \pi^{2}} m_{3 / 2}, \\
M_{3}=-3 \frac{g_{3}^{2}}{16 \pi^{2}} m_{3 / 2} .
\end{gathered}
$$

Third generation soft SUSY breaking scalar squared masses are given by

$$
\begin{aligned}
m_{U_{3}}^{2}= & \left(-\frac{88}{25} g_{1}^{4}+8 g_{3}^{4}+2 f_{t} \hat{\beta}_{f_{t}}\right) \frac{m_{3 / 2}^{2}}{\left(16 \pi^{2}\right)^{2}}+m_{0}^{2}(3), \\
m_{D_{3}}^{2}= & \left(-\frac{22}{25} g_{1}^{4}+8 g_{3}^{4}+2 f_{b} \hat{\beta}_{f_{b}}\right) \frac{m_{3 / 2}^{2}}{\left(16 \pi^{2}\right)^{2}}+m_{0}^{2}(3), \\
m_{Q_{3}}^{2}= & \left(-\frac{11}{50} g_{1}^{4}-\frac{3}{2} g_{2}^{4}+8 g_{3}^{4}+f_{t} \hat{\beta}_{f_{t}}+f_{b} \hat{\beta}_{f_{b}}\right) \frac{m_{3 / 2}^{2}}{\left(16 \pi^{2}\right)^{2}} \\
& +m_{0}^{2}(3), \\
m_{L_{3}}^{2}= & \left(-\frac{99}{50} g_{1}^{4}-\frac{3}{2} g_{2}^{4}+f_{\tau} \hat{\beta}_{f_{\tau}}\right) \frac{m_{3 / 2}^{2}}{\left(16 \pi^{2}\right)^{2}}+m_{0}^{2}(3), \\
m_{E_{3}}^{2}= & \left(-\frac{198}{25} g_{1}^{4}+2 f_{\tau} \hat{\beta}_{f_{\tau}}\right) \frac{m_{3 / 2}^{2}}{\left(16 \pi^{2}\right)^{2}}+m_{0}^{2}(3),
\end{aligned}
$$

while first/second generation scalar squared masses are given by similar formulas but where the associated Yukawa couplings may be safely ignored and the bulk sfermion mass is changed from $m_{0}^{2}(3) \rightarrow m_{0}^{2}(1,2)$.

For soft SUSY breaking Higgs masses, we propose (in accord with Ref. [2]) that each Higgs doublet receives an independent bulk mass contribution so that

$$
\begin{aligned}
m_{H_{u}}^{2}= & \left(-\frac{99}{50} g_{1}^{4}-\frac{3}{2} g_{2}^{4}+3 f_{t} \hat{\beta}_{f_{t}}\right) \frac{m_{3 / 2}^{2}}{\left(16 \pi^{2}\right)^{2}}+m_{H_{u}}^{2}(\text { bulk }), \\
m_{H_{d}}^{2}= & \left(-\frac{99}{50} g_{1}^{4}-\frac{3}{2} g_{2}^{4}+3 f_{b} \hat{\beta}_{f_{b}}+f_{\tau} \hat{\beta}_{f_{\tau}}\right) \frac{m_{3 / 2}^{2}}{\left(16 \pi^{2}\right)^{2}} \\
& +m_{H_{d}}^{2}(\text { bulk }) .
\end{aligned}
$$

The freedom of independent bulk Higgs soft masses $m_{H_{u}}^{2}\left(\right.$ bulk) and $m_{H_{d}}^{2}($ bulk) may be traded using the electroweak minimization conditions for the alternative weak-scale inputs $\mu$ and $m_{A}$ (as in the two-extra-parameternon-universal Higgs model (NUHM2) SUSY model [39]).

Using this flexibility, we again scan over AMSB parameters as in Sec. I but now also including:

(i) $\mu: 100-500 \mathrm{GeV}$ and

(ii) $m_{A}: 0.25-10 \mathrm{TeV}$.

The results are plotted again in the $\Delta_{\mathrm{EW}}$ vs $m_{h}$ plane and shown in Fig. 2. From the figure, we see that now many 


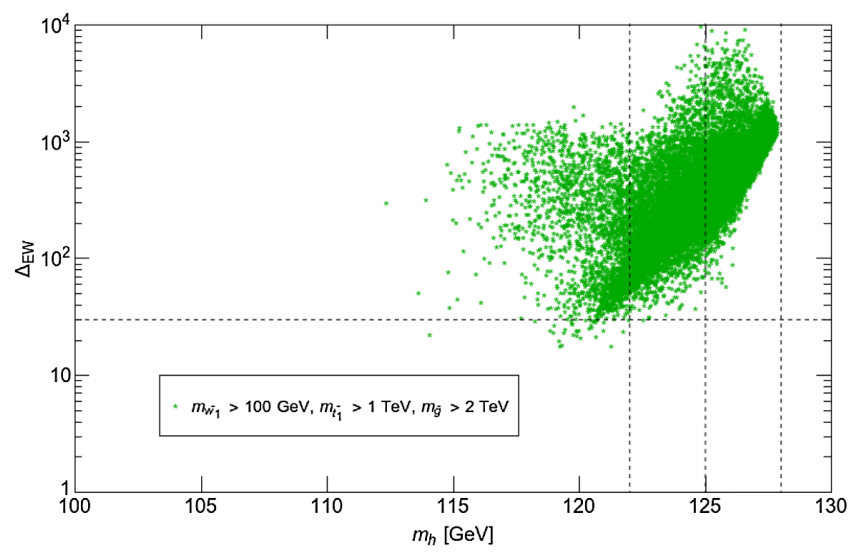

FIG. 2. Plot of points in the $\Delta_{\mathrm{EW}}$ vs $m_{h}$ plane from a scan over AMSB parameter space with added bulk Higgs soft terms but without bulk $A_{0}$ terms.

points have dropped into the natural area where $\Delta_{\mathrm{EW}}<30$. However, almost all these points also have $m_{h} \lesssim 122 \mathrm{GeV}$.

Thus, following Ref. [2], we propose adding as well a bulk contribution to the trilinear soft terms. Then, the $A$ parameters are given by

$$
\begin{gathered}
A_{t}=\frac{\hat{\beta}_{f_{t}}}{f_{t}} \frac{m_{3 / 2}}{16 \pi^{2}}+A_{0}, \\
A_{b}=\frac{\hat{\beta}_{f_{b}}}{f_{b}} \frac{m_{3 / 2}}{16 \pi^{2}}+A_{0}, \quad \text { and } \\
A_{\tau}=\frac{\hat{\beta}_{f_{\tau}}}{f_{\tau}} \frac{m_{3 / 2}}{16 \pi^{2}}+A_{0} .
\end{gathered}
$$

The quantities $\hat{\beta}_{f_{i}}$ that enter the expressions for scalar masses and $A$ parameters are given by the standard expressions

$$
\begin{aligned}
\hat{\beta}_{f_{t}}= & 16 \pi^{2} \beta_{t}=f_{t}\left(-\frac{13}{15} g_{1}^{2}-3 g_{2}^{2}-\frac{16}{3} g_{3}^{2}+6 f_{t}^{2}+f_{b}^{2}\right), \\
\hat{\beta}_{f_{b}} & =16 \pi^{2} \beta_{b} \\
& =f_{b}\left(-\frac{7}{15} g_{1}^{2}-3 g_{2}^{2}-\frac{16}{3} g_{3}^{2}+f_{t}^{2}+6 f_{b}^{2}+f_{\tau}^{2}\right),
\end{aligned}
$$$$
\hat{\beta}_{f_{\tau}}=16 \pi^{2} \beta_{\tau}=f_{\tau}\left(-\frac{9}{5} g_{1}^{2}-3 g_{2}^{2}+3 f_{b}^{2}+4 f_{\tau}^{2}\right) .
$$

The first two generations of squark and slepton masses are given by the corresponding formulas above with the Yukawa couplings set to zero. Equations (6)-(18) serve as renormalization group equation (RGE) boundary
TABLE I. Input parameters and masses in $\mathrm{GeV}$ units for two natural generalized anomaly mediation SUSY benchmark points with $m_{t}=173.2 \mathrm{GeV}$ using ISAJET7.88.

\begin{tabular}{lcc}
\hline \hline Parameter & nAMSB1 & nAMSB2 \\
\hline$m_{3 / 2}$ & 135,000 & 135,000 \\
$\tan \beta$ & 10 & 10 \\
$m_{0}(1,2)$ & 13000 & 5000 \\
$m_{0}(3)$ & 5000 & 5000 \\
$A_{0}$ & 5300 & 5990 \\
$\mu$ & 200 & 200 \\
$m_{A}$ & 2000 & 2000 \\
$m_{\tilde{g}}$ & 3022.0 & 2907.6 \\
$m_{\tilde{u}_{L}}$ & 13188.0 & 5516.4 \\
$m_{\tilde{u}_{R}}$ & 13277.2 & 5687.0 \\
$m_{\tilde{e}_{R}}$ & 12911.1 & 4757.4 \\
$m_{\tilde{t}_{1}}$ & 1446.4 & 1802.4 \\
$m_{\tilde{t}_{2}}$ & 3550.5 & 3940.6 \\
$m_{\tilde{b}_{1}}$ & 3582.3 & 3970.8 \\
$m_{\tilde{b}_{2}}$ & 5083.5 & 5415.8 \\
$m_{\tilde{\tau}_{1}}$ & 4675.1 & 4685.4 \\
$m_{\tilde{\tau}_{2}}$ & 4929.0 & 5026.1 \\
$m_{\tilde{\nu}_{\tau}}$ & 4901.2 & 5031.8 \\
$m_{\tilde{W}_{2}}$ & 409.9 & 401.1 \\
$m_{\tilde{W}_{1}}$ & 196.6 & 196.9 \\
$m_{\tilde{Z}_{4}}$ & 1272.5 & 1259.0 \\
$m_{\tilde{Z}_{3}}$ & 418.1 & 410.0 \\
$m_{\tilde{Z}_{2}}$ & 209.6 & 209.7 \\
$m_{\tilde{Z}_{1}}$ & 185.3 & 185.3 \\
$m_{h}$ & 124.7 & 124.7 \\
$\Omega_{\tilde{Z}_{1}}^{\text {std }} h^{2}$ & 0.009 & 0.009 \\
$B F(b \rightarrow s \gamma) \times 10^{4}$ & 3.2 & 3.3 \\
$B F\left(B_{s} \rightarrow \mu^{+} \mu^{-}\right) \times 10^{9}$ & 3.8 & 3.8 \\
$\sigma^{\mathrm{SI}}\left(\tilde{Z}_{1}, p\right)(\mathrm{pb})$ & $9.0 \times 10^{-9}$ & $9.5 \times 10^{-9}$ \\
$\sigma^{\mathrm{SD}}\left(\tilde{Z}_{1} p\right)(\mathrm{pb})$ & $2.1 \times 10^{-4}$ & $2.1 \times 10^{-4}$ \\
$\left.\langle\sigma v\rangle\right|_{v \rightarrow 0}\left(\mathrm{~cm}{ }^{3} / \mathrm{sec}\right)$ & $2.7 \times 10^{-25}$ & $2.7 \times 10^{-25}$ \\
$\Delta_{\mathrm{EW}}$ & 10.3 & 23.7 \\
\hline \hline & & \\
\hline & & \\
\hline
\end{tabular}

conditions at $Q=m_{\mathrm{GUT}}$. The nAMSB model is therefore characterized by the parameter set,

$m_{0}(1,2), \quad m_{0}(3), \quad m_{3 / 2}, \quad A_{0}, \quad \tan \beta, \quad \mu$, and $m_{A}$.

This generalized AMSB model has been encoded into ISAJET7.88 [34] for spectra calculations and collider event generation. For greater generality, we have also allowed for split generations with $m_{0}(1,2) \neq m_{0}(3)$. This feature, however, is not necessary for bringing AMSB models into accord with naturalness and with the measured value of the Higgs mass. Two advantages of split generations include the following:

(1) Heavy first/second generation bulk contributions soften the SUSY flavor and $C P$ problems via decoupling [40]. 

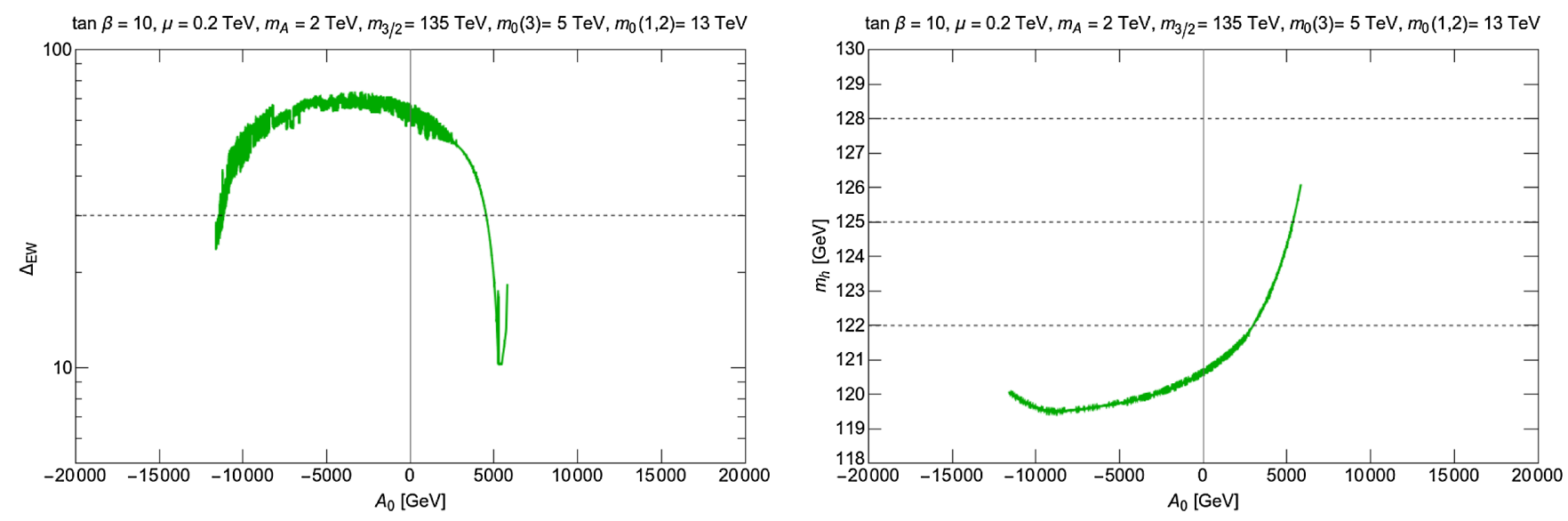

FIG. 3. Frame (a): $\Delta_{\mathrm{EW}}$ vs $A_{0}$ for $m_{3 / 2}=135 \mathrm{TeV}, m_{0}(1,2)=13 \mathrm{TeV}, m_{0}(3)=5 \mathrm{TeV}, \mu=200 \mathrm{GeV}$, and $m_{A}=2000 \mathrm{GeV}$. In frame (b), we plot $m_{h}$ vs $A_{0}$ for the same parameters.

(2) They actually act, via two-loop RGE terms, to further suppress third generation soft terms bring the nAMSB model into even greater accord with naturalness [as exhibited by the two benchmark points in Table I where the first (nAMSB1) adopts $m_{0}(1,2) \gg m_{0}(3)$ and where the second (nAMSB2) adopts $\left.m_{0}(1,2)=m_{0}(3)\right]$.

To see the effect of including the bulk $A_{0}$ trilinear soft term, we adopt a nAMSB benchmark point with parameters $m_{3 / 2}=135 \mathrm{TeV}, \quad m_{0}(1,2)=13 \mathrm{TeV}, \quad m_{0}(3)=5 \mathrm{TeV}$, $\mu=200 \mathrm{GeV}$, and $m_{A}=2 \mathrm{TeV}$ with $\tan \beta=10$. In Fig. 3(a), we show the value of $\Delta_{\mathrm{EW}}$ as we vary $A_{0}$. For no bulk trilinear, with $A_{0}=0$, then $\Delta_{\mathrm{EW}} \sim 70$, and the model requires electroweak (EW) fine-tuning at the $1.4 \%$ level. As $A_{0}$ varies and becomes large positive or negative, then large mixing in the stop sector leads to a reduction in both $\Sigma_{u}^{u}\left(\tilde{t}_{1,2}\right)$ values. For $A_{0} \sim+5 \mathrm{TeV}$, then $\Delta_{\mathrm{EW}}$ drops to as low as 10. In frame Fig. 3(b), we show the variation in $m_{h}$ vs $A_{0}$. With no bulk contribution to $A$ terms, $m_{h} \sim 120 \mathrm{GeV}$. As $A_{0}$ increases to $\sim+5 \mathrm{TeV}$, then the added stop mixing increases $m_{h}$ until it reaches the $\sim 125 \mathrm{GeV}$ level.

In Fig. 4, we show the nAMSB spectra plot from our benchmark point where now we adopt $A_{0}=+5.3 \mathrm{TeV}$. From the plot, we see that the $W, Z$, and $h$ are clustered around the $\sim 100 \mathrm{GeV}$ scale with the Higgsinos $\tilde{W}_{1}^{ \pm}$and $\tilde{Z}_{1,2}$ clustered not too far away at $\sim 200 \mathrm{GeV}$ as required by naturalness. Meanwhile, first/second generation matter sfermions lie in the multi-TeV range at $\sim 13 \mathrm{TeV}$. For the gauginos, we have $m_{\tilde{g}} \sim 3 \mathrm{TeV}$, well beyond current LHC limits which at present require $m_{\tilde{g}} \gtrsim 2 \mathrm{TeV}$. What is characteristic about nAMSB is the rather light winos $\tilde{W}_{2}^{ \pm}$ and $\tilde{Z}_{3}$ with mass $\sim 400 \mathrm{GeV}$. The bino $\tilde{Z}_{4}$ has mass

\footnotetext{
${ }^{7}$ These spectra are rather similar to that expected by Dine from the intermediate branch of the IIB string theory landscape [41].
}

$\sim 1.2 \mathrm{TeV}$. For the top squarks, we find them to be highly mixed by the large $A_{t}$ term with $m_{\tilde{t}_{1}} \sim 1.4 \mathrm{TeV}$ and $m_{\tilde{t}_{2}} \sim 3.5 \mathrm{TeV}$. As we shall see, the nAMSB mass spectrum leads to very different expectations for LHC signatures as compared to the old mAMSB model.

The precise benchmark point mass values are listed numerically in Table I along with various calculated dark matter and $B$-decay observables. For this point, the thermal WIMP abundance of Higgsino-like WIMP comes in (from ISARED [42]) at $\Omega_{\tilde{Z}_{1}}^{T P} h^{2} \sim 0.009$, a factor 13.3 below the measured abundance. In the case of nAMSB, we also expect the presence of a SUSY-DFSZ axion which would likely make up the remaining dark matter abundance. A complete calculation requires an eight-coupled Boltzmann equation computation [43]. The WIMP detection rates are also given, but in this case, they must be scaled down by factors of $\xi \equiv \Omega_{\tilde{Z}_{1}} h^{2} / 0.12$ for the SI and spin-dependent (SD) direct detection rates. For the IDD detection rate, the Higgsino-like WIMPs mainly annihilate into the $W W$

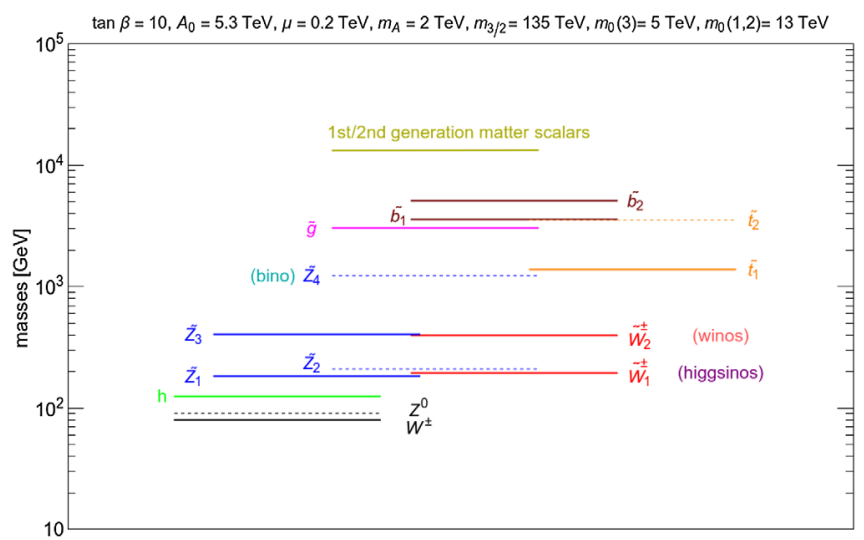

FIG. 4. A typical superparticle mass spectrum generated from nAMSB as in Table I for point nAMSB1. 


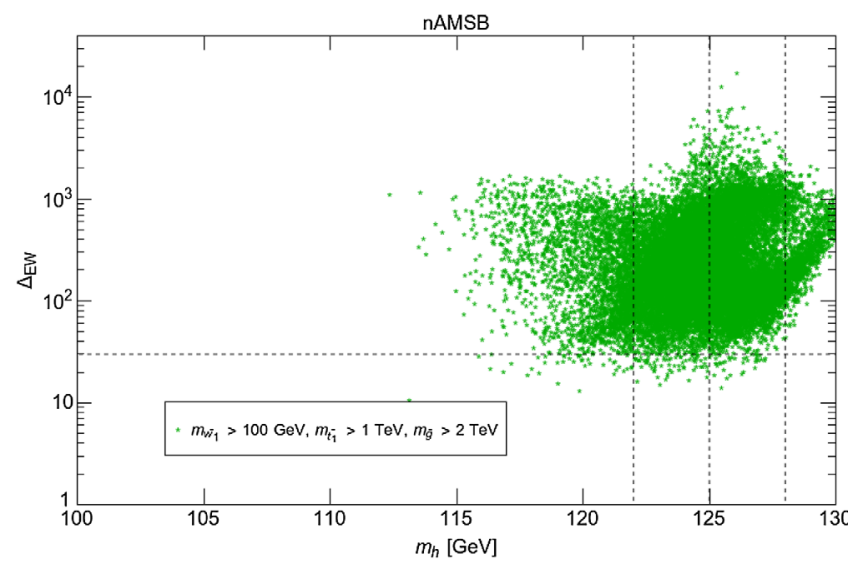

FIG. 5. Plot of points from a scan over nAMSB parameter space in the $\Delta_{\mathrm{EW}}$ vs $m_{h}$ plane.

channel but in this case must be scaled down by a factor $\xi^{2}$. These rescalings, due to diminished WIMP number density, bring the detection rates near or below current experimental limits (see Ref. [19] for a recent summary). The naturalness parameter for the benchmark point lies at $\Delta_{\mathrm{EW}}=10.3$, so the model is quite natural with just $\sim 10 \%$ EW fine-tuning required.

In Fig. 5, we repeat the above AMSB parameter space scans except now we include as well a scan over

$$
A_{0}:-20 \rightarrow+20 \mathrm{TeV} .
$$

From the figure, we now see data points in accord with LHC sparticle mass constraints which populate the $\Delta_{\mathrm{EW}}<30$ naturalness regime while also allowing for $m_{h} \sim 125 \pm 3 \mathrm{GeV}$. Thus, the combination of independent bulk Higgs masses and an added bulk trilinear soft term $A_{0}$ allows us to bring the AMSB model into accord with LHC Higgs mass measurements and naturalness requirements and dark matter constraints.

\section{B. nAMSB model line}

In phenomenological studies of models for new physics, it is frequently useful to adopt model lines wherein new particle masses increase in a controlled manner, thus allowing for collider reach calculations [44], decoupling, etc. We may elevate our previous benchmark model to a model line by allowing the gravitino mass to float so all sparticle masses increase with $m_{3 / 2}$ from the LHC limits until they become unnatural or decouple.

In Fig. 6, we show three frames resulting from a nAMSB model line vs $m_{3 / 2}$ starting at $m_{3 / 2} \simeq 80 \mathrm{TeV}$. This latter value corresponds to $m_{\tilde{g}} \sim 2 \mathrm{TeV}$, just beyond the current LHC limits from simplified models [35]. In frame (a), we show how $\Delta_{\mathrm{EW}}$ varies. At lower values $m_{3 / 2} \sim$ $100-150 \mathrm{TeV}, \Delta_{\mathrm{EW}} \sim 10$, and the model is highly natural. As $m_{3 / 2}$ increases, all soft terms increase according to
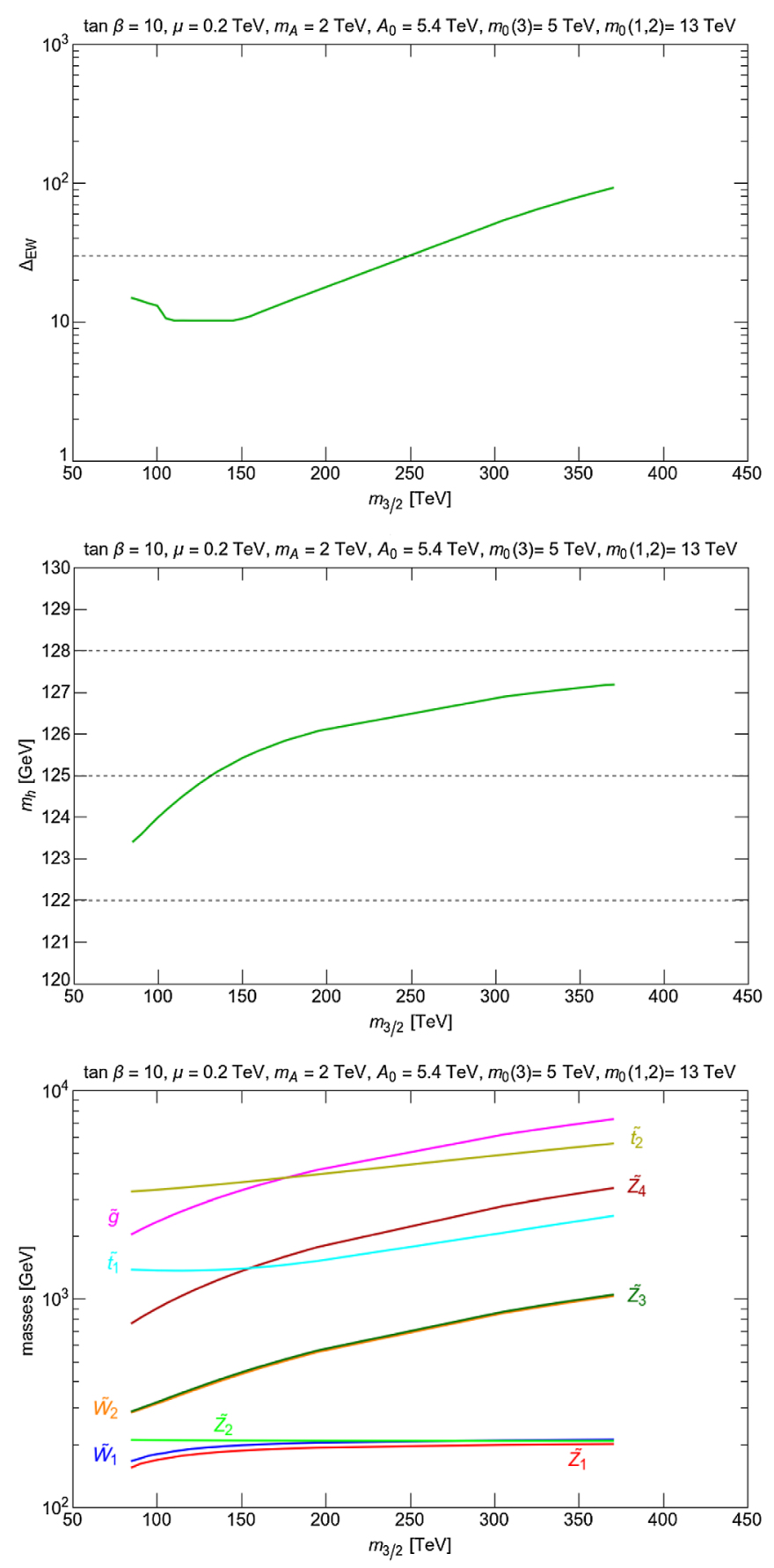

FIG. 6. Plot of (a) $\Delta_{\mathrm{EW}}$, (b) $m_{h}$, and (c) various sparticle masses vs $m_{3 / 2}$ along a nAMSB model line with $m_{0}(1,2)=13 \mathrm{TeV}$, $m_{0}(3)=5 \mathrm{TeV}, A_{0}=5.3 \mathrm{TeV}, m_{A}=2 \mathrm{TeV}$, and $\mu=200 \mathrm{GeV}$ with $\tan \beta=10$.

Eqs. (6)-(18). As $m_{3 / 2}$ increases to the vicinity of $250 \mathrm{TeV}$, $\Delta_{\mathrm{EW}}$ moves beyond the 30 value where fine-tuning begins to be required in the weak-scale scalar potential. Thus, the regime where $m_{3 / 2} \lesssim 250 \mathrm{TeV}$ seems favored from a naturalness perspective. In frame (b), we show the corresponding value of $m_{h}$ along the nAMSB model line. Its value begins at $m_{h} \sim 124 \mathrm{GeV}$ for $m_{3 / 2} \sim 80 \mathrm{TeV}$ 
and increases to $\sim 127 \mathrm{GeV}$ for $m_{3 / 2}$ as high as $370 \mathrm{TeV}$. Thus, the light Higgs mass stays within its required range (allowing for $\sim \pm 2 \mathrm{GeV}$ theory error in our $m_{h}$ calculation) over the entire model line. In frame (c), we show various sparticle masses along the model line. The Higgsinos $\tilde{W}_{1}^{ \pm}$and $\tilde{Z}_{1,2}$ remain clustered at $\sim 200 \mathrm{GeV}$ since the $\mu$ parameter remains fixed. The gluinos and stops lie in the several $\mathrm{TeV}$ range, and as their masses increase, so, too, do the radiative corrections $\sum_{u}^{u}\left(\tilde{t}_{1,2}\right)$ in Eq. (5). Over the range of $m_{3 / 2}$ consistent with naturalness, $m_{\tilde{g}}$ varies from 2 to $4 \mathrm{TeV}$, while the lighter stop is in the range $m_{\tilde{t}_{1}} \sim 1.3-1.5 \mathrm{TeV}$. Of considerable interest for collider searches is the range of the wino masses $m_{\tilde{W}_{2}}^{ \pm}$and $m_{\tilde{Z}_{3}}$. These vary from $300 \mathrm{GeV}$ for $m_{3 / 2} \sim 100 \mathrm{TeV}$ to $\sim 600 \mathrm{GeV}$ for $m_{3 / 2} \sim 250 \mathrm{GeV}$. This will have important ramifications for the discussion of collider searches in the next section.

\section{Locus of natural AMSB parameters}

It is important to check from scans over the full generalized AMSB parameter space in Eq. (22) where exactly the natural solutions with low $\Delta_{\text {EW }}$ exist. Thus, here, we implement a scan over the full parameter space and plot each parameter vs $\Delta_{\mathrm{EW}}$. To aid the reader, we show the demarcation where $\Delta_{\text {EW }}$ exceeds 30 , although it is simple to extract parameter locales for other choices of a maximal $\Delta_{\mathrm{EW}}$ value.

In Fig. 7(a), we show $\Delta_{\text {EW }}$ vs $m_{3 / 2}$ from our scan. Our points are extracted from the general scan with limits given above and also from a dedicated scan over parameters where $\Delta_{\text {EW }}$ is more likely to be $\lesssim 30: m_{3 / 2} \sim 80-300 \mathrm{GeV}$, $\mu: 100-350 \mathrm{GeV}$, and $A_{0}: 0.5 m_{0}(3)-2 m_{0}(3)$. All points have $122 \mathrm{GeV}<m_{h}<128 \mathrm{GeV}$. From frame (a), we see that to maintain naturalness $m_{3 / 2}$ is roughly bounded from above by about $300 \mathrm{GeV}$ (in accord with the above nAMSB model line).

In frame (b), we show $\Delta_{\mathrm{EW}}$ vs $m_{0}(1,2)$. The first and second generation scalar masses enter the naturalness measure via electroweak $D$-term contributions [26,45], and these terms tend to cancel for nearly degenerate matter scalars. Thus, a wide range of $m_{0}(1,2)$ values extending up into the $10-20 \mathrm{TeV}$ range is allowed by naturalness. Such large first/second generation matter scalar masses allow for at least a partial decoupling solution to the SUSY flavor and $C P$ problem (which may rearise with the addition of flavordependent bulk soft terms).

In Fig. 7(c), we show $\Delta_{\mathrm{EW}}$ vs $m_{0}(3)$. In this case, an upper bound of $m_{0}(3) \lesssim 8 \mathrm{TeV}$ emerges. This is because for too large values of third generation matter scalars the $\Sigma_{u}^{u}\left(\tilde{t}_{1,2}\right)$ contributions become large, thus requiring some electroweak fine-tuning.

In Fig. 8(a), we show $\Delta_{\text {EW }}$ vs $A_{0} / m_{0}(3)$. Here, we see that for $A_{0} \sim 0, \Delta_{\mathrm{EW}}$ is always $\gtrsim 30$ and unnatural. For $A_{0} / m_{0}(3) \sim-2$, then $\Delta_{\mathrm{EW}}$ drops below 30. This occurs
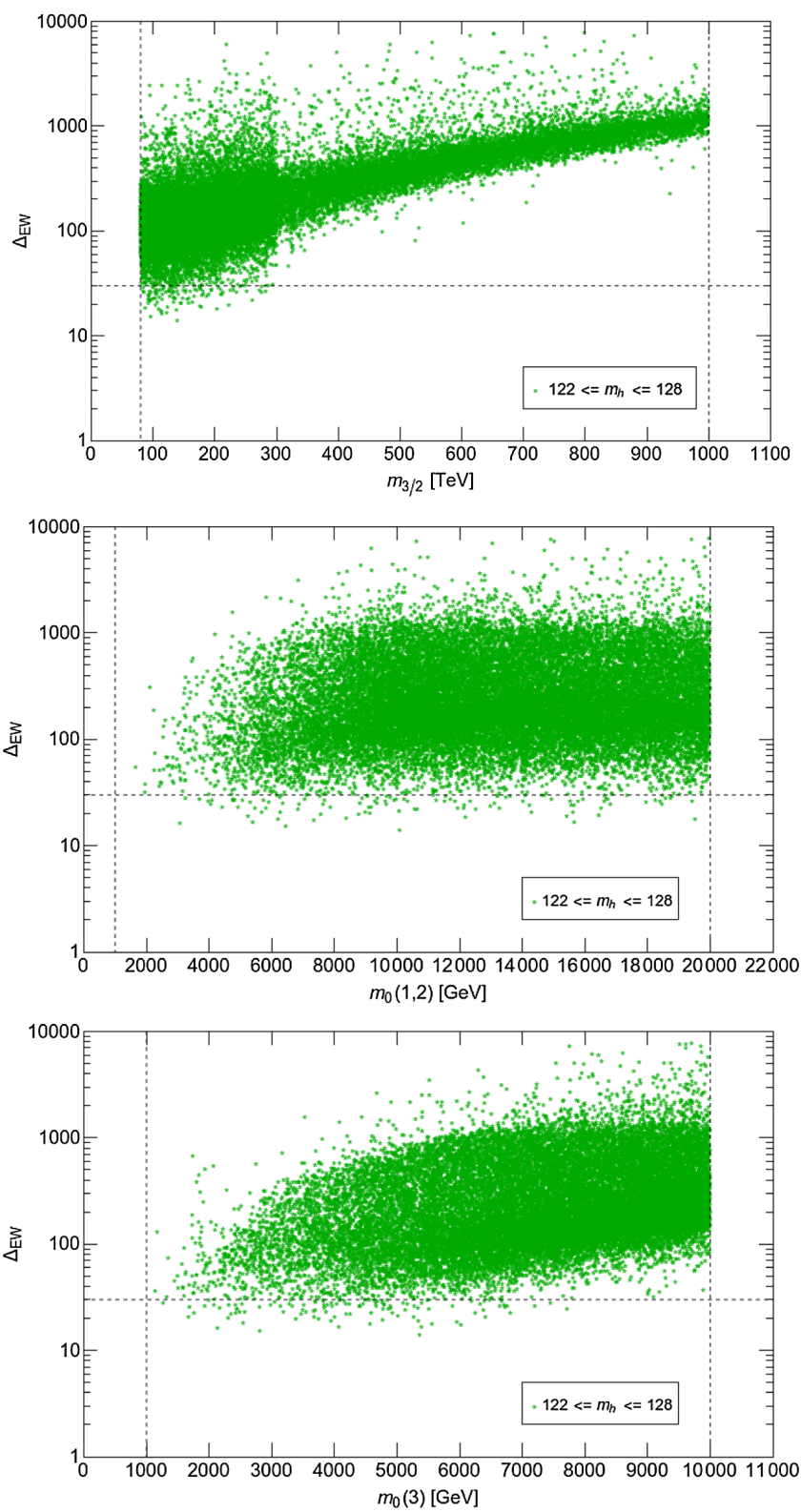

FIG. 7. Plot of nAMSB parameter scan in the $\Delta_{\mathrm{EW}}$ vs (a) $m_{3 / 2}$, (b) $m_{0}(1,2)$ and (c) $m_{0}(3)$ planes. The greater density of points for $m_{3 / 2} \lesssim 300 \mathrm{TeV}$ comes from the narrow scan added to the broad scan.

even more sharply for $A_{0} / m_{0}(3) \sim+1$. As noted previously, the large $A_{0}$ values decrease the $\Sigma_{u}^{u}\left(\tilde{t}_{1,2}\right)$ contributions while lifting $m_{h} \sim 125 \mathrm{GeV}$ [25]. Frame (b) shows $\mu$ vs $\Delta_{\mathrm{EW}}$. Here, we see a sharp demarcation for naturalness when $\mu \lesssim 350 \mathrm{GeV}$, the lighter the better. This is also seen from direct computation from Eq. (5). In frame (c), we show variation vs $\tan \beta$. In this case, a wide range of $\tan \beta$ is allowed by naturalness, but not the very highest values where $\tan \beta \gtrsim 40$. For such high $\tan \beta$, then the $\Sigma_{u}^{u}\left(\tilde{b}_{1,2}\right)$ may become large, thus requiring some fine-tuning. In frame (d), we show variation with $m_{A}$. In this case, for $m_{A} \gg m_{Z}$, then $m_{H_{d}} \sim m_{A}$, and naturalness in Eq. (5) 

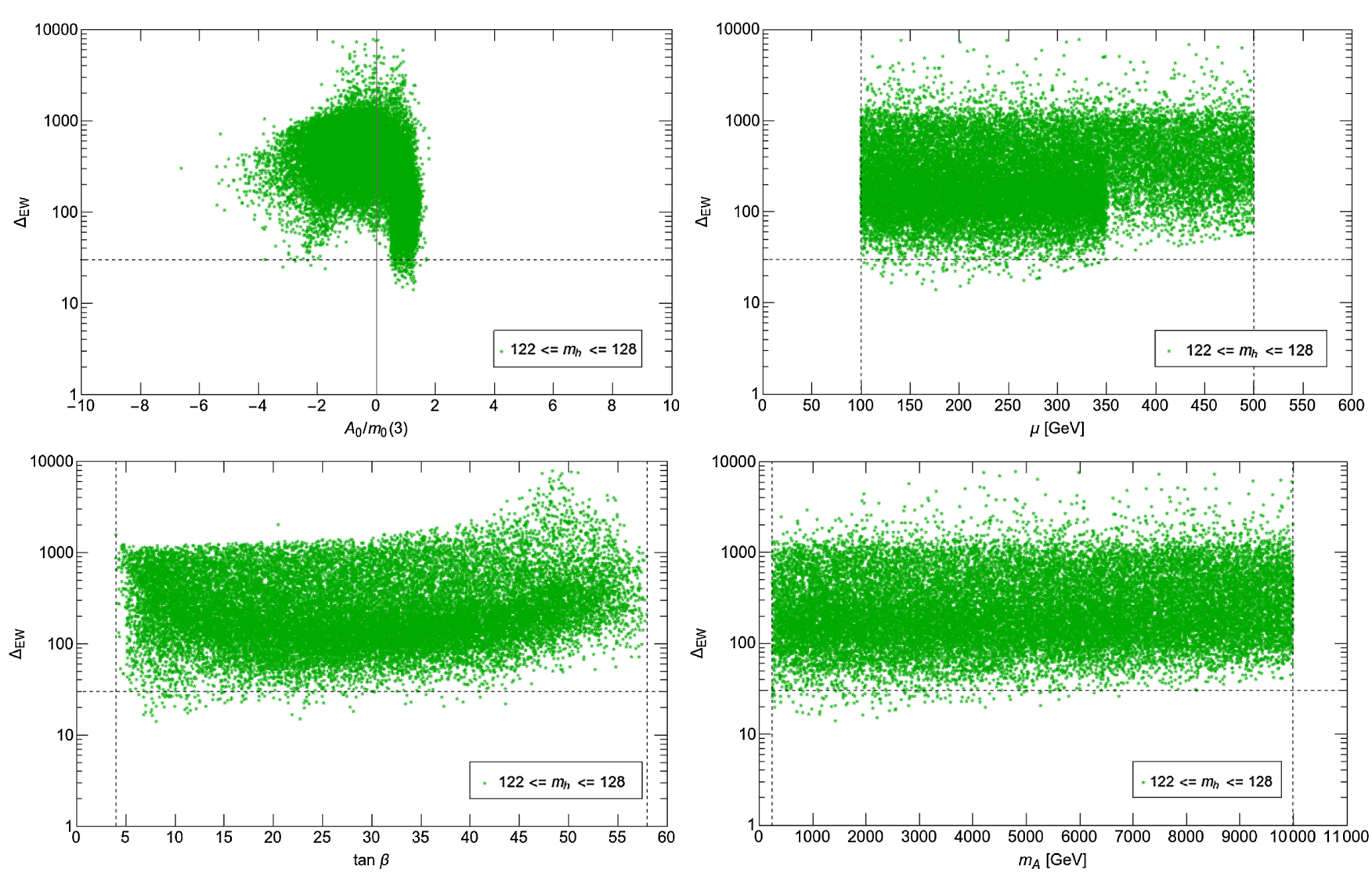

FIG. 8. Plot of nAMSB parameter scan in the $\Delta_{\mathrm{EW}}$ vs (a) $A_{0} / m_{0}(3)$, (b) $\mu$, (c) $\tan \beta$, and (d) $m_{A}$ planes.

would require $m_{A} / \tan \beta \lesssim \sqrt{30\left(m_{Z}^{2} / 2\right)}$. This requires $m_{A}$ to be bounded from above by about $7-8 \mathrm{TeV}$.

\section{Bounds on sparticle masses in the natural AMSB model}

It is desirable in any SUSY model to extract upper bounds on various sparticle masses from naturalness in order to establish a testability criterion for the model. Thus, in this section, we implement the full scan over nAMSB parameter space (as delineated above).

In Fig. 9, we show $\Delta_{\text {EW }}$ vs $m_{\tilde{g}}$. Here, we see that $m_{\tilde{g}}$ ranges from the LHC lower limit of $\sim 2 \mathrm{TeV}$ up to $m_{\tilde{g}} \sim$ $6 \mathrm{TeV}$ before the model becomes unnatural (where $\Delta_{\mathrm{EW}}$ exceeds $\sim 30$ ). The expected range in $m_{\tilde{g}}$ will of course have important implications for gluino searches at present and planned hadron colliders. The upper bound $m_{\tilde{g}} \lesssim 6 \mathrm{TeV}$ is in accord with other SUSY models: gravity mediation in NUHM2 $[26,31,46]$ and in mirage mediation [47]. The reason is that $m_{\tilde{g}}$ feeds into the RG evolution of top-squark soft terms and a larger value of $m_{\tilde{g}}$ therefore increases the $\Sigma_{u}^{u}\left(\tilde{t}_{1,2}\right)$ values.

In Fig. 10, we show the expected range for top-squark masses $m_{\tilde{t}_{1}}$ [frame (a)] and $m_{\tilde{t}_{2}}$ [frame (b)]. In frame (a), we see that $m_{\tilde{t}_{1}}$ ranges from its approximate LHC lower bound of $m_{\tilde{t}_{1}} \gtrsim 1 \mathrm{TeV}$ up to at most $3 \mathrm{TeV}$ before the nAMSB model becomes unnatural. Meanwhile, from frame (b), we see that $m_{\tilde{t}_{2}}$ can range up to $\sim 6 \mathrm{TeV}$.

In Fig. 11, we plot the expected range of wino mass $m_{\tilde{W}_{2}}$. In this case, $m_{\tilde{W}_{2}}$ (which is $\simeq m_{\tilde{Z}_{3}}$ ) ranges from a lower bound $\sim 250 \mathrm{GeV}$ to an upper bound from naturalness of $m_{\tilde{W}_{2}} \sim 800 \mathrm{GeV}$. In AMSB models, the weak-scale wino mass is typically $m$ (wino) $\sim m_{\tilde{g}} / 8$ so that the wino mass upper bound arises due to the $m_{\tilde{g}}$ limits arising from $\Sigma_{u}^{u}\left(\tilde{t}_{1,2}\right)$. The wino mass range will also have important consequences for collider signatures for nAMSB.

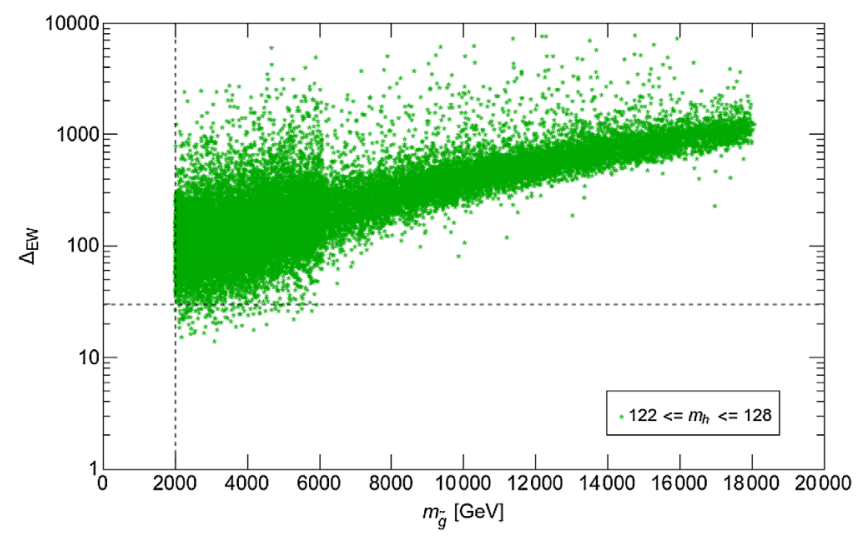

FIG. 9. Plot of nAMSB parameter scan in the $\Delta_{\mathrm{EW}}$ vs $m_{\tilde{g}}$ plane. 

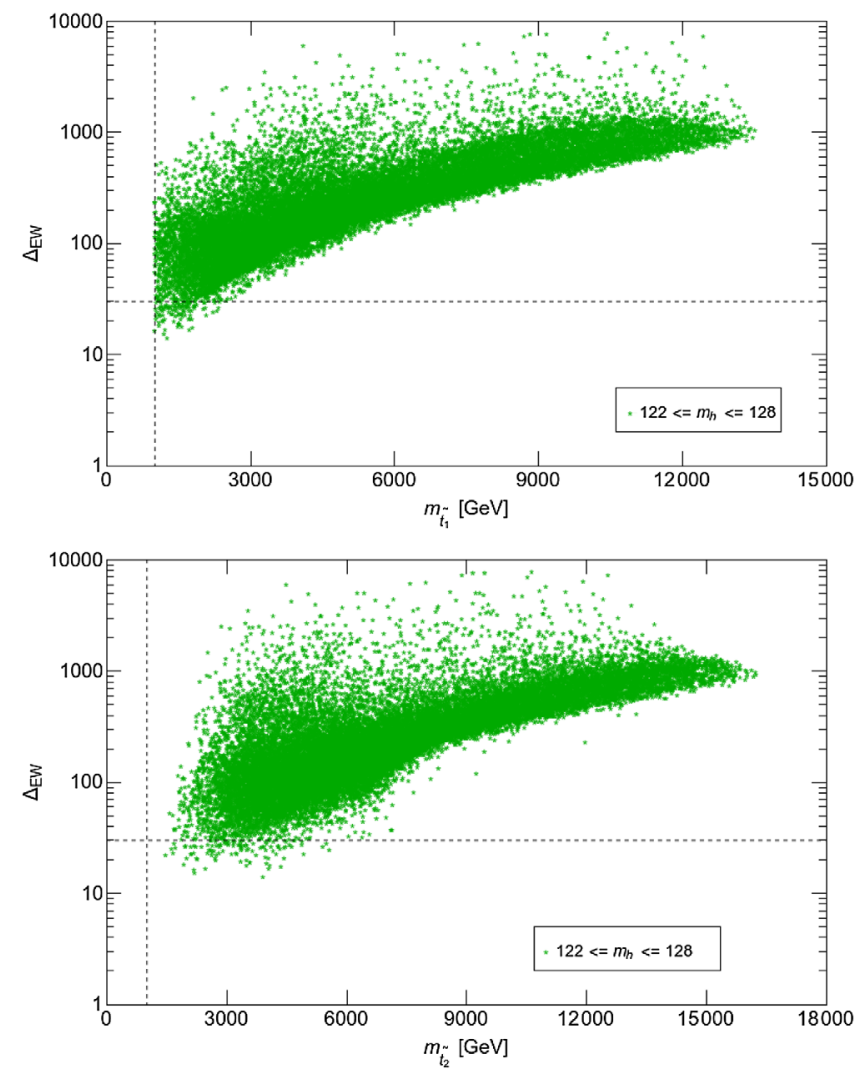

FIG. 10. Plot of nAMSB parameter scan in the $\Delta_{\mathrm{EW}}$ vs (a) $m_{\tilde{t}_{1}}$ and (b) $m_{\tilde{t}_{2}}$ planes.

Lastly, we plot the phenomenologically important mass gap $m_{\tilde{Z}_{2}}-m_{\tilde{Z}_{1}}$ vs $\Delta_{\mathrm{EW}}$ in Fig. 12. This mass gap enters Higgsino pair production signatures at both the LHC and at linear $e^{+} e^{-}$colliders. Due to the proximity of the winos to the Higgsinos, the mass gap is expected to be larger than in models with unified gaugino masses. Indeed, from the figure, we see that $m_{\tilde{Z}_{2}}-m_{\tilde{Z}_{1}}$ ranges from about $10 \mathrm{GeV}$ all the way up to $100 \mathrm{GeV}$. This may be compared to models with gaugino mass unification where instead $m_{\tilde{Z}_{2}}-m_{\tilde{Z}_{1}}$ ranges from $\sim 10-25 \mathrm{GeV}$ typically [26].

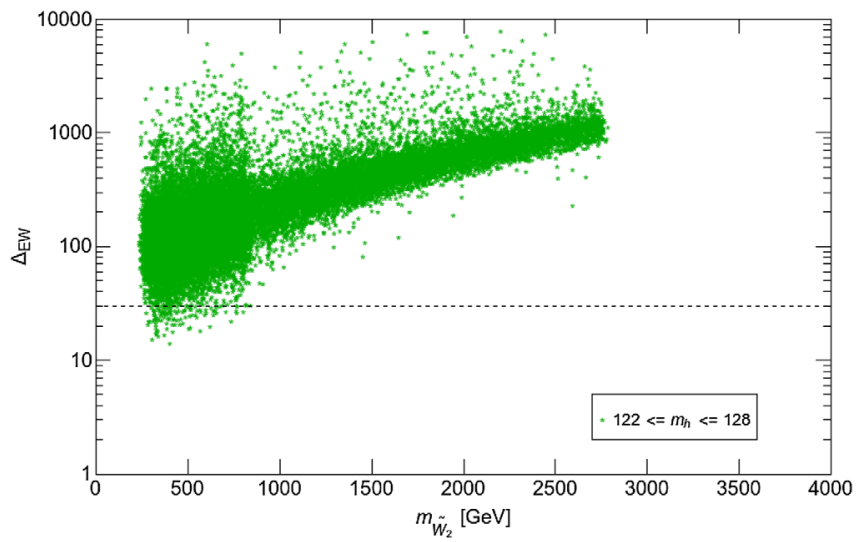

FIG. 11. Plot of nAMSB parameter scan in the $\Delta_{\mathrm{EW}}$ vs $m_{\tilde{W}_{2}}$ plane.

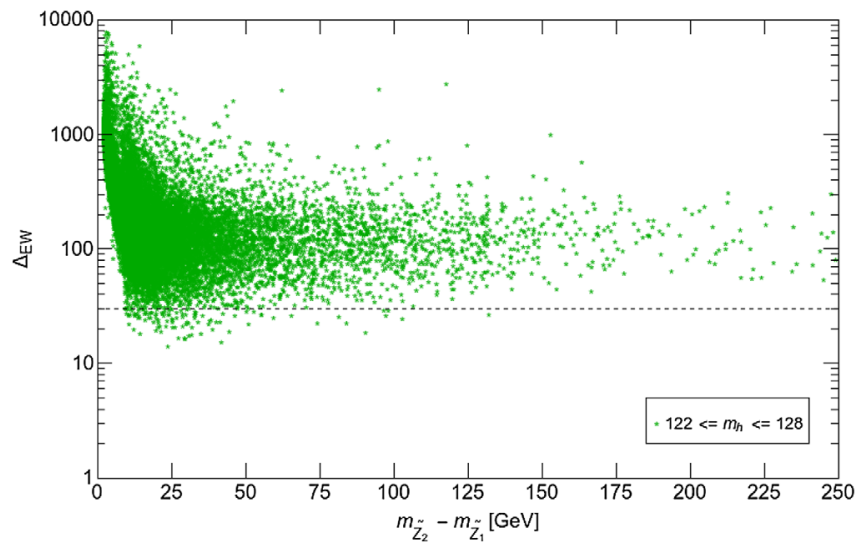

FIG. 12. Plot of nAMSB parameter scan in the $\Delta_{\mathrm{EW}}$ vs $m_{\tilde{Z}_{2}}-$ $m_{\tilde{Z}_{1}}$ plane.

\section{CONSEQUENCES FOR COLLIDER AND DARK MATTER SEARCHES}

One of the many intriguing aspects of the mAMSB model is that it led to rather unique collider signaturessuch as the presence of quasistable winos in sparticle cascade decays. In this section, we will find very different collider signatures for the nAMSB model.

\section{A. LHC}

\section{Gluino pair production}

At the CERN LHC, an important SUSY search channel comes from gluino pair production. In nAMSB, almost always $m_{\tilde{g}}>m_{\tilde{t}_{1}}$ so that $\tilde{g} \rightarrow \tilde{t}_{1} \bar{t}, \tilde{t}_{1}^{*} t$ followed by $\tilde{t}_{1} \rightarrow$ $t \tilde{Z}_{1,2,3}$ or $b \tilde{W}_{1,2}$. Thus, gluino pair production events are expected to be rich in both $t$ and $b$ quarks arising from gluino cascade decays. Recently, the reach for various LHC luminosity upgrades has been estimated for natural SUSY models. It is found in Ref. [48] that high-luminosity Large Hadron Collider (HL-LHC) with $\sim 3 \mathrm{ab}^{-1}$ of integrated luminosity has a $5 \sigma$ reach in $m_{\tilde{g}}$ to about $m_{\tilde{g}} \sim 2.8 \mathrm{TeV}$. From Fig. 9, we see that this covers only a small portion of nAMSB parameter space.

Meanwhile, the reach of high-energy Large Hadron Collider (HE-LHC) has also been estimated. Using $\sqrt{s}=$ $33 \mathrm{TeV}$ and $1 \mathrm{ab}^{-1}$ integrated luminosity, it is found that the HE-LHC reach extends to about $5 \mathrm{TeV}[47,49]$, thus covering essentially all of nAMSB parameter space. Updated run parameters for the HE-LHC have recently been proposed as $\sqrt{s}=27 \mathrm{TeV}$ but $L=10-15 \mathrm{ab}^{-1}$. The HE-LHC reach using the lower energy/higher luminosity parameter is likely comparable to our quoted numbers.

\section{Top squark pair production}

Top squark pair production $p p \rightarrow \tilde{t}_{1} \tilde{t}_{1}^{*}$ is another important LHC search channel. In nAMSB, we expect $m_{\tilde{t}_{1}}: 1-3 \mathrm{TeV}$. This is to be compared to the $5 \sigma \mathrm{HL}-\mathrm{LHC}$ 
reach to $m_{\tilde{t}_{1}} \sim 1.2 \mathrm{TeV}$ [50]. Thus, in this channel again, the HL-LHC will be able to cover only a small portion of mAMSB parameter space. The $5 \sigma$ HE-LHC reach extends to $m_{\tilde{t}_{1}} \sim 3.2 \mathrm{TeV}$ [47]. Thus, HE-LHC should be able to cover essentially all mAMSB parameter space via topsquark pair searches.

\section{Higgsino pair production}

From Eq. (5), we find that for $\Delta_{\mathrm{EW}}<30$, then $m_{\tilde{Z}_{1.2}}$, $m_{\tilde{W}_{1}} \sim \mu \lesssim 350 \mathrm{GeV}$. Thus, Higgsino pair production reactions occur at potentially observable rates [51] at the LHC. Typically, most of the energy from Higgsino pair production goes into making up the two $\tilde{Z}_{1}$ particles' rest masses, so the visible energy release is small, making Higgsino pair production reactions challenging to see $[33,52]$. A way forward has been proposed in Refs. [53], where one produces $\tilde{Z}_{1} \tilde{Z}_{2}$ in association with hard initial state jet radiation. Then, one may trigger on the hard jet $\left(\right.$ or $\mathbb{E}_{T}$ ) and within such events search for low mass, soft opposite-sign dileptons arising from $\tilde{Z}_{2} \rightarrow \tilde{Z}_{1} \ell^{+} \ell^{-}$decay. Recent search results from CMS have been presented [54], and results from ATLAS are imminent [55]. With HL-LHC, this channel may well be able to explore the entire parameter space. A distinctive feature of the nAMSB model is that the $\tilde{Z}_{2}-\tilde{Z}_{1}$ mass gap is expected to be substantially larger than in models with gaugino mass unification or in mirage mediation [56] due to the smaller Higgsino-wino mass gap.

\section{Wino pair production}

In SUSY models with light Higgsinos, a compelling new signature has emerged [57]: wino pair production followed by decay to same-sign dibosons (SSdB): $p p \rightarrow \tilde{W}_{2}^{ \pm} \tilde{Z}_{3}$ with $\tilde{W}_{2} \rightarrow W \tilde{Z}_{1,2}$ and $\tilde{Z}_{3} \rightarrow W^{ \pm} \tilde{W}_{1}^{\mp}$. The Higgsinos at the end of the decay chain are again quasivisible, so one really expects half the time a $W^{ \pm} W^{ \pm}+\mathbb{E}_{T}$ signal which has very low Standard Model (SM) backgrounds arising mainly from $t \bar{t} W$ and other processes. Signal and background have been estimated in Refs. [51,57,58]. It is found that the reach of HL-LHC extends to about $m_{\tilde{W}_{2}} \lesssim 1 \mathrm{TeV}$. Thus, in this channel as well, we expect HL-LHC to completely cover the nAMSB parameter space. If such a signal does not emerge at HL-LHC, then the mAMSB model will be ruled out. If a signal does emerge, then in Ref. [58], several suggestions have been proposed to extract a measurement of the wino masses: via counting, via distributions, and via ++ to -- charge asymmetry. The importance of this channel for the nAMSB model derives from the expected weak-scale gaugino mass ratio in AMSB models $M_{1}: M_{2}: M_{3} \sim 0.4: 0.13: 1$ where winos are expected to be far lighter than gluinos (or binos).

\section{B. Linear electron-positron colliders}

Since (simple) natural SUSY models require the presence of light Higgsinos [via Eq. (5) and Fig. 8(b)], then the proposed International Linear $e^{+} e^{-}$Collider
(ILC), is expected to become a Higgsino factory for $\sqrt{s}>2 m$ (Higgsino [59]. The main production reactions are $e^{+} e^{-} \rightarrow \tilde{W}_{1}^{+} \tilde{W}_{1}^{-}$and $\tilde{Z}_{1} \tilde{Z}_{2}$. In spite of the low energy release expected from these reactions, the clean operating environment and low SM backgrounds should allow the Higgsino pair production events to be easily visible. These features, along with kinematic restrictions on the events, should allow for precision mass measurements of $\tilde{W}_{1}, \tilde{Z}_{1}$, and $\tilde{Z}_{2}$. If the ILC is built with extendable energy ranging up to $\sqrt{s} \sim 1 \mathrm{TeV}$, then there is a strong chance that direct wino production could also be detected via the $e^{+} e^{-} \rightarrow$ $\tilde{W}_{1}^{ \pm} \tilde{W}_{2}^{\mp}$ channel in nAMSB.

Since the mass gaps $m_{\tilde{W}_{1}}-m_{\tilde{Z}_{1}}$ and $m_{\tilde{Z}_{2}}-m_{\tilde{Z}_{1}}$ depend sensitively on the Higgsino-gaugino mixing, it has been shown in Refs. [59-61] that the electroweak gaugino masses can also be extracted to percent-level accuracy. Once the EW gaugino masses are known to sufficient accuracy, then they may be run via RGEs to higher energies to test whether or not they unify. In the case of nAMSB, where $M_{2} \ll M_{1}$ is expected, the ILC would be able to quickly show that anomaly mediation is the likely underlying SUSY model.

\section{Dark matter: WIMPs and axions}

Dark matter in nAMSB is expected to be a Higgsino-like WIMP plus the SUSY DFSZ axion admixture, as with other natural SUSY models. As seen in Table I, the $\tilde{Z}_{1}$ are thermally underproduced in the early Universe although nonthermal processes such as axino and/or saxion production and decay in the early Universe may augment these rates. The remaining abundance is expected to be comprised of axions. In the case where thermal WIMP production dominates, then indeed the bulk of dark matter would be axions. Precise estimates of the dark matter abundance require the solution of eight coupled Boltzmann equations which track: (1) the radiation density and number densities for (2) WIMPs, (3) thermal- and (4) coherent oscillation production of axions, (5) axinos, (6) thermaland (7) coherent oscillation-production of saxions and (8) gravitino production [43].

To assess WIMP detection prospects, one must account for the diminished abundance of WIMPs that is quantified by $\xi \equiv \Omega_{\tilde{Z}_{1}} h^{2} / 0.12$ and where in Table I we would expect $\xi$ as low as 0.075 . The spin-independent neutralino-proton scattering cross section from IsaTOOLS is shown in Table I. Mutiplying by $\xi$ and comparing to recent exclusion limits, the benchmark point is found to be slightly excluded by recent LUX limits. But for the case of the nAMSB model, we expect typically higher $\sigma^{\mathrm{SI}}\left(\tilde{Z}_{1} p\right)$ rates because the WIMP-WIMP- $h$ coupling, which enters the SI detection rate, is a product of gaugino times the Higgsino component. The typically reduced wino mass in nAMSB (as compared to models with gaugino mass unification) raises the scattering rate somewhat. Detailed WIMP scattering 
calculations in this model will be needed for a complete assessment of detectability. ${ }^{8}$ The $\sigma^{\mathrm{SD}}$ rate and indirect detection rate (in terms of $\left.\langle\sigma v\rangle\right|_{v \rightarrow 0}$ ) are also given. Multiplying by $\xi$ and $\xi^{2}$ respectively, these two rates are still below current bounds as shown in Ref. [19].

While our benchmark point is nominally excluded, even with the inclusion of the $\xi$ factor, we remark that further entropy dumping in the early Universe could possibly lower the WIMP abundance even further from its thermal value [63]. A perhaps more compelling scenario is that the nAMSB model may provide a viable niche for light axino dark matter. In usual gravity mediation, the axino (and saxion) is expected to gain masses of order $\sim m_{3 / 2}[64,65]$. In nAMSB, we would expect the saxion to gain a bulk soft mass $m_{s} \sim 1 \mathrm{TeV}$, but the axino mass could be suppressed, leading to an unstable lightest neutralino which suffers late decay to, e.g., $\tilde{a}+\gamma, Z, h$. In such a case, dark matter would be an axion/axino admixture.

Meanwhile, detection of the SUSY DFSZ axion has been shown to be more difficult than in the non-SUSY models due to the circulation of Higgsinos in the $a-\gamma-\gamma$ triangle coupling [66]. Thus, we do not expect detection of the associated axion any time soon unless the presence of exotic matter in the $a-\gamma-\gamma$ coupling leads to an increased axion detection rate for microwave cavity experiments.

\section{CONCLUDING REMARKS}

In this paper, we have proposed a new anomaly-mediation paradigm model which evades the problems of 1) too low a value of $m_{h}, 2$ ) unnaturalness, and 3) winolike LSPs which may be excluded by a lack of IDD of dark matter. Our new model, dubbed natural anomaly-mediated SUSY breaking or nAMSB, merely incorporates the inclusion of nonuniversal bulk scalar masses and a bulk trilinear term $A_{0}$. The former allows for small $\mu$ as required by naturalness and leads instead to a Higgsino-like WIMP as LSP. The inclusion of a bulk $A_{0}$ term allows for large stop mixing

\footnotetext{
${ }^{8}$ Recently, a detailed study of WIMP detection in nAMSB was made [62]. In that paper, it is shown that nAMSB WIMPs which make up all of dark matter would be excluded by direct and indirect WIMP detection experiments. However, a substantial amount of nAMSB model parameter space is still intact for the case of mixed axion-WIMP dark matter where the WIMPs only make up a small portion of the total dark matter density.
}

which lifts $m_{h}$ up to $\sim 125 \mathrm{GeV}$ while decreasing the topsquark radiative corrections to the scalar potential $\Sigma_{u}^{u}\left(\tilde{t}_{1,2}\right)$. In fact, these revision were suggested by the model's creators [2].

We computed the sparticle mass spectrum in nAMSB. While weak-scale gaugino masses are still related as $M_{1}: M_{2}: M_{3} \sim 0.4: 0.13: 1$ leading to the wino as the lightest gaugino, the lightest charginos and neutralinos are instead mainly Higgsino-like (but with a non-negligible wino component). These modifications bring the model in line with Higgs mass and naturalness constraints and also in line with dark matter constraints [62] for the case of mixed axion-WIMP dark matter (while the case of WIMP-only nAMSB dark matter seems to be excluded). But the modifications also greatly alter the collider and dark matter signatures which are usually expected from anomaly mediation. Instead of quasistable charged winos leading to terminating tracks in collider experiments, now there are more rapidly decaying Higgsinos at the bottom of the spectra. We computed upper bounds on gluino and topsquark masses in nAMSB and found these to be possibly well beyond the reach of HL-LHC although they should be accessible to HE-LHC. However, since Higgsinos are required to be not too far from the $100 \mathrm{GeV}$ scale, then the $\ell^{+} \ell^{-} j+\mathbb{E}_{T}$ signature should likely be accessible to HL-LHC albeit with larger chargino and neutralino mass gaps than in models with unified gauginos. Also, the SSdB signature from wino pair production should be detectable over the entire natural range of wino masses in nAMSB leading to a conclusive test of this model. An ILC operating with $\sqrt{s}>2 m$ (Higgsino) could also discover SUSY and unravel the underlying mediation mechanism via precision Higgsino pair production measurements.

Dark matter is expected to consist of a Higgsino-like WIMP plus axion admixture. Prospects for WIMP detection should be better than in natural models with gaugino mass unification due to the presence of rather light winos which enhance the SI DD scattering rates. Axions may remain difficult to detect. A further alternative is that the nAMSB model may provide a viable home for mixed axion/axino dark matter.

\section{ACKNOWLEDGMENTS}

This work was supported in part by the US Department of Energy, Office of High Energy Physics.
[1] J. Polchinski, Phys. Rev. Lett. 75, 4724 (1995).

[2] L. Randall and R. Sundrum, Nucl. Phys. B557, 79 (1999).

[3] I. Jack, D. R. T. Jones, and A. Pickering, Phys. Lett. B 426, 73 (1998); L. V. Avdeev, D. I. Kazakov, and I. N. Kondrashuk,
Nucl. Phys. B510, 289 (1998); G. F. Giudice, M. A. Luty, H. Murayama, and R. Rattazzi, J. High Energy Phys. 12 (1998) 027; J. A. Bagger, T. Moroi, and E. Poppitz, J. High Energy Phys. 04 (2000) 009; A. Pomarol and R. Rattazzi, 
J. High Energy Phys. 05 (1999) 013; M. K. Gaillard and B. D. Nelson, Nucl. Phys. B588, 197 (2000); P. Binetruy, M. K. Gaillard, and B. D. Nelson, Nucl. Phys. B604, 32 (2001); M. Dine and N. Seiberg, J. High Energy Phys. 03 (2007) 040; S. P. de Alwis, Phys. Rev. D 77, 105020 (2008); M. Dine and P. Draper, J. High Energy Phys. 02 (2014) 069; F. D'Eramo, J. Thaler, and Z. Thomas, J. High Energy Phys. 06 (2012) 151.

[4] J. L. Feng, T. Moroi, L. Randall, M. Strassler, and S. f. Su, Phys. Rev. Lett. 83, 1731 (1999).

[5] T. Gherghetta, G. F. Giudice, and J. D. Wells, Nucl. Phys. B559, 27 (1999).

[6] J. L. Feng and T. Moroi, Phys. Rev. D 61, 095004 (2000).

[7] H. Baer, J. K. Mizukoshi, and X. Tata, Phys. Lett. B 488, 367 (2000).

[8] A. J. Barr, C. G. Lester, M. A. Parker, B. C. Allanach, and P. Richardson, J. High Energy Phys. 03 (2003) 045; B. C. Allanach, T. J. Khoo, and K. Sakurai, J. High Energy Phys. 11 (2011) 132.

[9] H. Baer, C. Kao, and J. Sayre, Phys. Rev. D 85, 035021 (2012).

[10] E. Bagnaschi et al., Eur. Phys. J. C 77, 268 (2017).

[11] Z. Chacko, M. A. Luty, I. Maksymyk, and E. Ponton, J. High Energy Phys. 04 (2000) 001; E. Katz, Y. Shadmi, and Y. Shirman, J. High Energy Phys. 08 (1999) 015; I. Jack and D. R. T. Jones, Phys. Lett. B 482, 167 (2000); M. Carena, K. Huitu, and T. Kobayashi, Nucl. Phys. B592, 164 (2001); R. Dermisek, H. Verlinde, and L. T. Wang, Phys. Rev. Lett. 100, 131804 (2008); M. Hindmarsh and D. R. T. Jones, Phys. Rev. D 87, 075022 (2013).

[12] F. Gabbiani, E. Gabrielli, A. Masiero, and L. Silvestrini, Nucl. Phys. B477, 321 (1996).

[13] G. Aad et al. (ATLAS Collaboration), J. High Energy Phys. 01 (2013) 131.

[14] T. Moroi and L. Randall, Nucl. Phys. B570, 455 (2000).

[15] A. Arbey, M. Battaglia, A. Djouadi, F. Mahmoudi, and J. Quevillon, Phys. Lett. B 708, 162 (2012); A. Arbey, A. Deandrea, and A. Tarhini, J. High Energy Phys. 05 (2011) 078.

[16] H. Baer, V. Barger, and A. Mustafayev, J. High Energy Phys. 05 (2012) 091.

[17] A. Arbey, A. Deandrea, F. Mahmoudi, and A. Tarhini, Phys. Rev. D 87, 115020 (2013).

[18] H. Baer, V. Barger, D. Mickelson, and M. PadeffkeKirkland, Phys. Rev. D 89, 115019 (2014).

[19] H. Baer, V. Barger, and H. Serce, Phys. Rev. D 94, 115019 (2016).

[20] M. L. Ahnen et al. (MAGIC and Fermi-LAT Collaborations), J. Cosmol. Astropart. Phys. 02 (2016) 039.

[21] H. Abdallah et al. (HESS Collaboration), Phys. Rev. Lett. 117, 111301 (2016).

[22] T. Cohen, M. Lisanti, A. Pierce, and T. R. Slatyer, J. Cosmol. Astropart. Phys. 10 (2013) 061.

[23] J. Fan and M. Reece, J. High Energy Phys. 10 (2013) 124.

[24] K. J. Bae, H. Baer, A. Lessa, and H. Serce, Front. Phys. 3, 49 (2015).

[25] H. Baer, V. Barger, P. Huang, A. Mustafayev, and X. Tata, Phys. Rev. Lett. 109, 161802 (2012).

[26] H. Baer, V. Barger, P. Huang, D. Mickelson, A. Mustafayev, and X. Tata, Phys. Rev. D 87, 115028 (2013).
[27] J. Ellis, K. Enqvist, D. Nanopoulos, and F. Zwirner, Mod. Phys. Lett. A 01, 57 (1986); R. Barbieri and G. Giudice, Nucl. Phys. B306, 63 (1988).

[28] R. Kitano and Y. Nomura, Phys. Rev. D 73, 095004 (2006); M. Papucci, J. T. Ruderman, and A. Weiler, J. High Energy Phys. 09 (2012) 035; C. Brust, A. Katz, S. Lawrence, and R. Sundrum, J. High Energy Phys. 03 (2012) 103.

[29] H. Baer, V. Barger, and D. Mickelson, Phys. Rev. D 88, 095013 (2013).

[30] A. Mustafayev and X. Tata, Indian J. Phys. 88, 991 (2014).

[31] H. Baer, V. Barger, and M. Savoy, Phys. Rev. D 93, 035016 (2016).

[32] K. L. Chan, U. Chattopadhyay, and P. Nath, Phys. Rev. D 58, 096004 (1998).

[33] H. Baer, V. Barger, and P. Huang, J. High Energy Phys. 11 (2011) 031.

[34] H. Baer, F. Paige, S. Protopopescu, and X. Tata, arXiv:hepph/0312045; H. Baer, C. H. Chen, R. B. Munroe, F. E. Paige, and X. Tata, Phys. Rev. D 51, 1046 (1995).

[35] M. Aaboud et al. (ATLAS Collaboration), Phys. Rev. D 97, 112001 (2018); T. Sakuma (CMS Collaboration), Proc. Sci., LHCP2016 (2017) 145.

[36] J. E. Kim and H. P. Nilles, Phys. Lett. 138B, 150 (1984).

[37] M. Dine, W. Fischler, and M. Srednicki, Phys. Lett. 104B, 199 (1981); A. R. Zhitnitsky, Yad. Fiz. 31, 497 (1980); [Sov. J. Nucl. Phys. 31, 260 (1980)].

[38] K. J. Bae, H. Baer, and E. J. Chun, Phys. Rev. D 89, 031701 (2014); K. J. Bae, H. Baer, and E. J. Chun, J. Cosmol. Astropart. Phys. 12 (2013) 028.

[39] D. Matalliotakis and H. P. Nilles, Nucl. Phys. B435, 115 (1995); M. Olechowski and S. Pokorski, Phys. Lett. B 344, 201 (1995); P. Nath and R. L. Arnowitt, Phys. Rev. D 56, 2820 (1997); J. Ellis, K. Olive, and Y. Santoso, Phys. Lett. B 539, 107 (2002); J. Ellis, T. Falk, K. Olive, and Y. Santoso, Nucl. Phys. B652, 259 (2003); H. Baer, A. Mustafayev, S. Profumo, A. Belyaev, and X. Tata, J. High Energy Phys. 07 (2005) 065.

[40] M. Dine, A. Kagan, and S. Samuel, Phys. Lett. B 243, 250 (1990); A. G. Cohen, D. B. Kaplan, and A. E. Nelson, Phys. Lett. B 388, 588 (1996); N. Arkani-Hamed and H. Murayama, Phys. Rev. D 56, R6733 (1997); H. Baer, A. Belyaev, T. Krupovnickas, and A. Mustafayev, J. High Energy Phys. 06 (2004) 044.

[41] M. Dine, J. High Energy Phys. 01 (2006) 162.

[42] H. Baer, C. Balazs, and A. Belyaev, J. High Energy Phys. 03 (2002) 042.

[43] K. J. Bae, H. Baer, A. Lessa, and H. Serce, J. Cosmol. Astropart. Phys. 10 (2014) 082.

[44] B. C. Allanach et al., Eur. Phys. J. C 25, 113 (2002).

[45] H. Baer, V. Barger, M. Padeffke-Kirkland, and X. Tata, Phys. Rev. D 89, 037701 (2014).

[46] H. Baer, V. Barger, and M. Savoy, Phys. Rev. D 93, 075001 (2016).

[47] H. Baer, V. Barger, J. S. Gainer, H. Serce, and X. Tata, Phys. Rev. D 96, 115008 (2017).

[48] H. Baer, V. Barger, J. S. Gainer, P. Huang, M. Savoy, D. Sengupta, and X. Tata, Eur. Phys. J. C 77, 499 (2017).

[49] H. Baer, V. Barger, J. S. Gainer, P. Huang, M. Savoy, H. Serce, and X. Tata, Phys. Lett. B 774, 451 (2017). 
[50] ATLAS Collaboration, CERN, Geneva, Technical Report No. ATL-PHYS-PUB-2013-011 (2013); CMS Collaboration, arXiv:1307.7135.

[51] H. Baer, V. Barger, P. Huang, D. Mickelson, A. Mustafayev, W. Sreethawong, and X. Tata, J. High Energy Phys. 12 (2013) 013; 06 (2015) 53.

[52] H. Baer, A. Mustafayev, and X. Tata, Phys. Rev. D 89, 055007 (2014).

[53] Z. Han, G. D. Kribs, A. Martin, and A. Menon, Phys. Rev. D 89, 075007 (2014); H. Baer, A. Mustafayev, and X. Tata, Phys. Rev. D 90, 115007 (2014); C. Han, D. Kim, S. Munir, and M. Park, J. High Energy Phys. 04 (2015) 132.

[54] CMS Collaboration, Report No. CMS-PAS-SUS-16-048.

[55] Talk by B. Hooberman, SUSY 2017 Meeting, Mumbai, India, 2017 (unpublished).

[56] H. Baer, V. Barger, H. Serce, and X. Tata, Phys. Rev. D 94, 115017 (2016).

[57] H. Baer, V. Barger, P. Huang, D. Mickelson, A. Mustafayev, W. Sreethawong, and X. Tata, Phys. Rev. Lett. 110, 151801 (2013).
[58] H. Baer, V. Barger, J. S. Gainer, M. Savoy, D. Sengupta, and X. Tata, Phys. Rev. D 97, 035012 (2018).

[59] H. Baer, V. Barger, D. Mickelson, A. Mustafayev, and X. Tata, J. High Energy Phys. 06 (2014) 172.

[60] H. Baer, M. Berggren, K. Fujii, S. L. Lehtinen, J. List, T. Tanabe, and J. Yan, Proc. Sci., ICHEP2016 (2016) 156; S. L. Lehtinen, H. Baer, M. Berggren, K. Fujii, J. List, T. Tanabe, and J. Yan, Proc. Sci., EPS-HEP2017 (2017) 306.

[61] K. Fujii et al., arXiv:1702.05333.

[62] H. Baer, V. Barger, D. Sengupta, and X. Tata, arXiv: 1803.11210.

[63] K. J. Bae, H. Baer, and A. Lessa, J. Cosmol. Astropart. Phys. 04 (2013) 041.

[64] E. J. Chun and A. Lukas, Phys. Lett. B 357, 43 (1995).

[65] J.E. Kim and M. S. Seo, Nucl. Phys. B864, 296 (2012).

[66] K. J. Bae, H. Baer, and H. Serce, J. Cosmol. Astropart. Phys. 06 (2017) 024. 